\title{
الطلبة الفصحاء في الكلام باللغة العربية من منظور علم المعاني \\ (دراسة وصفية في معهد دار العلوم بانيوأنيار الاسلامي باميكاسان)
}

\author{
Nurul Hadi \\ (IAIN Madura/nurulhadi@gmail.com)
}

\begin{abstract}
The most representative indicator of the success of Arabic learning process is the fluency of the students' speaking by using Arabic. This study aims to determine whether there are students who are fluent in spoken Arabic, to identify the students' characteristics who are categorically fluent in spoken Arabic, to identify how students gain fluency in spoken Arabic based on Ma'ani science perspective. This is a descriptive qualitative study, the data collected by using interviews, questionnaires, and observation. The data sources were the preachers/teachers and the students who were active and had been active in the Arabic Language Center of Darul Ulum Islamic Boarding School of Banyuanyar. The results of this study revealed: First, students who are fluent in spoken Arabic were classified as fluent after studying Arabic for four months at the Arabic Language Center. Second, there are four indicators of students to be classified as fluent Arabic speakers, the first and the most representative indicator is the proficiency of students in Arabic debate contest in various themes, the second is students' ability to converse using Arabic in many different situations, the third is the ability to talk using the original Arabic, and the fourth is the students' ability in a Arabic speech. The way the students gain fluency in spoken Arabic is dependent on the personality of each student. The diligent students tend to have high spirit in practicing conversational Arabic, acquiring and learning Arabic, and also supported by strong intelligence, then they will acquire fluency in speaking Arabic.
\end{abstract}

\section{Keywords:}

Fluency; Ma’ani science; Arabic; Islamic boarding school

DOI: $10.19105 /$ ojbs.v13i1.2273

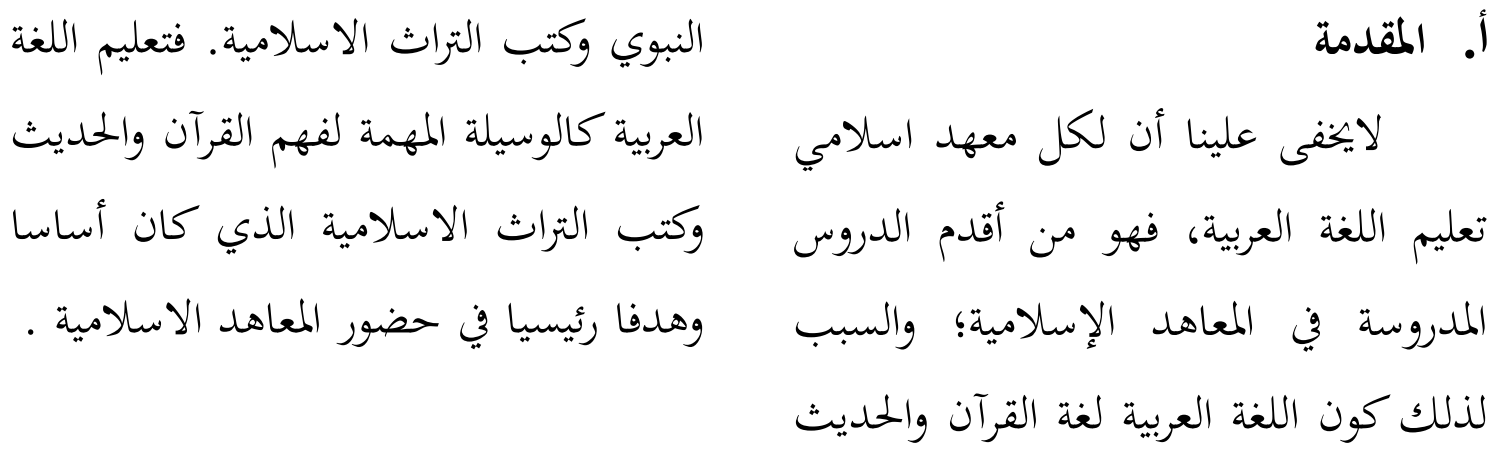


ثم تطور تعليم اللغة العربية من دور إلى حيث لا تقتصر في النصوص الدينية كما

أدوار، سواء كان من جهة هدف التعليم أو مرت.

فكل من الهدف والمواد التعليمي للغة

مواده أو وسائله أو طرقه في المهاء هدراه

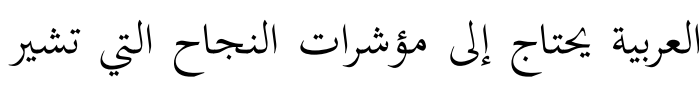

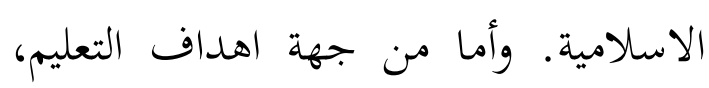

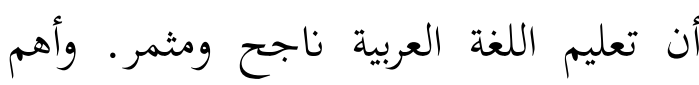

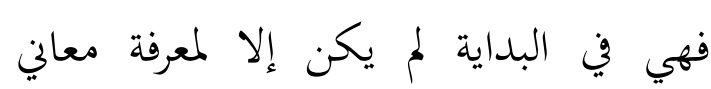

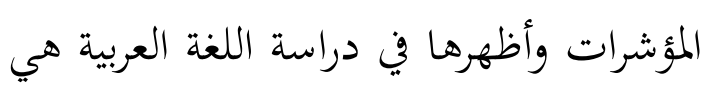

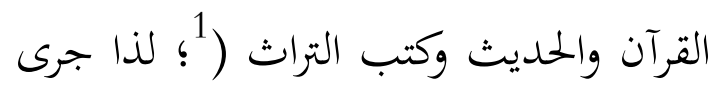
قدرة الطلبة في الأداء بالكلام باللغة العربية تعليم اللغة العربية ملتزما بتعليم القرآن أو في التعبير الشفهي بها؛ لأنه كالوسيلة والحديث وكتب التراث جنبا بجنب. ثم تطور البارزة لمعرفة ما في الطلبة من الكفاية اللغوية هدف التعليم من بجرد فهم النصوص العربية

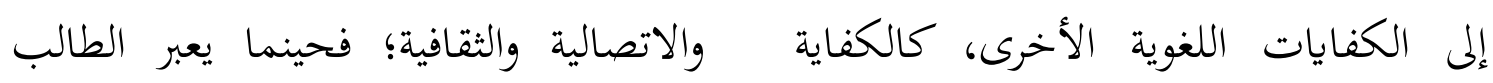

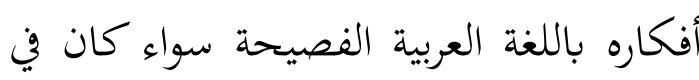
اللغوية والكفاية الثقافية والكفاية الاتصالية

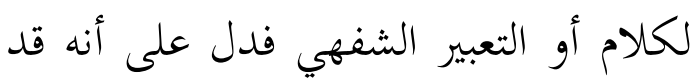

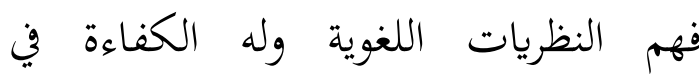
المهارات اللغوية وفي نفس الوقت أنه يشير النيات إلى معرفته في الثقافة العربية.

انطلاقا من أهمية الفصاحة في الكلام

والتعبير الشفهي كمؤشر النجاح في تعلم 2). والذي دل على ذلك تطور مواد التعليم في المعاهد حيث استخدام كتاب المحاورة لحسن باهرون وكتاب اللغة العربية للناشئين وكتاب اللغة العربية بين يديك وغيره في

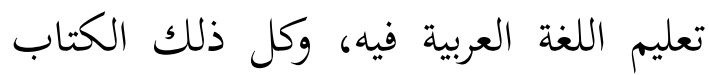
يستهدف إلى ثلاث الكفايات السابقة. اللغة العربية فيتبادر في ذهن الباحث وفي العصور السابقة كانت مواد تعليم السؤال: هل توجد الصفة الفصيحة عند اللغة العربية هي القرآن والحمديث وكتب الطلبة في تعلم اللغة العربية بمنظور علم التراث نفسها، ثم تطورت وتوسعت إلى المعاني في المعاهد الاسلامي؟ وهذا السؤال ينطلق من هموم الباحث نظرا إلى أن المعاهد موضوعات أخرى من موضوعات الحياة

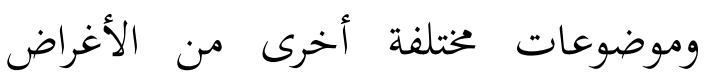

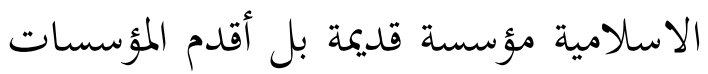
في إندونيسيا التي تعلم اللغة العربية. وفي

${ }^{1}$ Ahmad Fuad Effendy, "Metodologi Pengajaran Bahasa Arab", Malang: Misykat, 2005, 7.

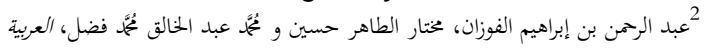

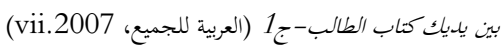


جانب آخر أن نظرية الفصاحة في علم العربية". وهدف البحث هو تسليط الضوء

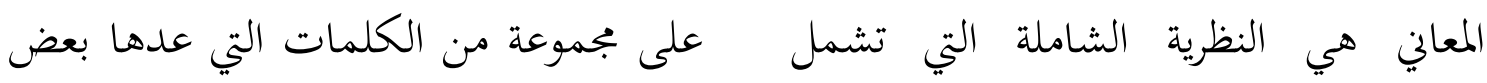

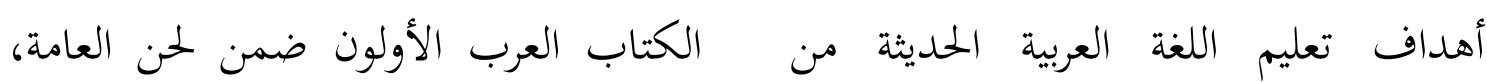

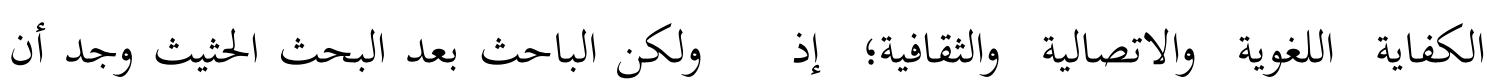

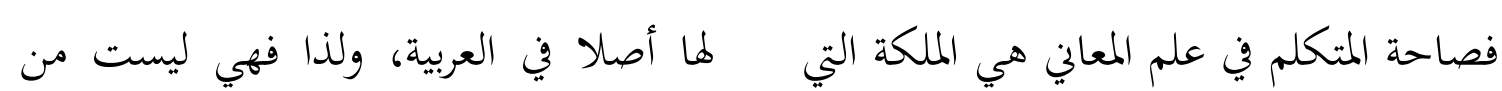

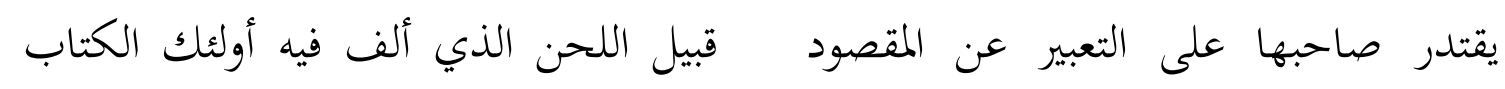
بكلام فصيح في أي غرض كان 3.

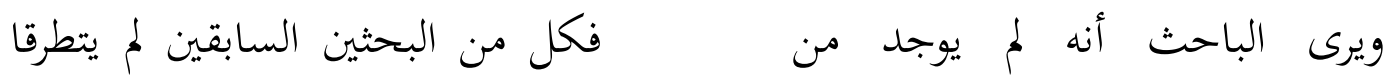
الباحثين من يتطرق في هذا المجال خاصة في م عن عن مواصفات الفصاحة عند الدارسين للغة

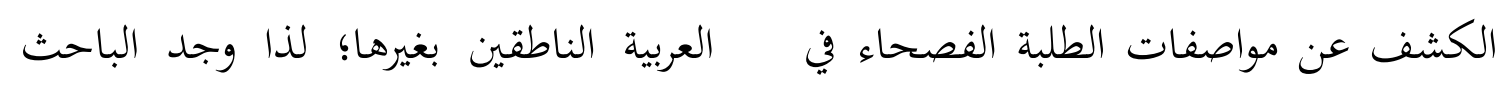
الكلام باللغة العربية في المعاهد الاسلامية. أهمية قوية لهذه الدراسة. ومما ذكر من خلفيات الدراسة وضع الباحث أسئلة البحث الآتية: (1) هل من رغم ذلك هناك بعض الدراسات السابقة

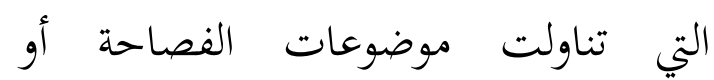

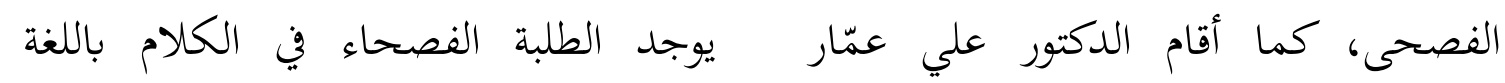

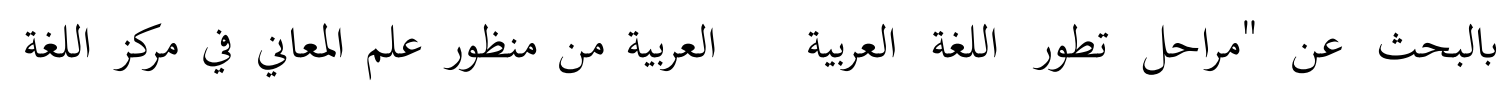

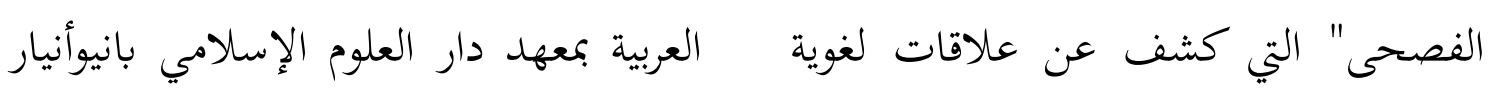

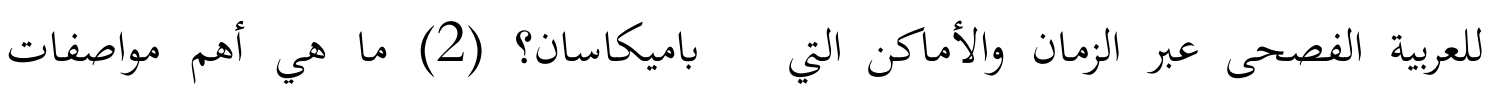

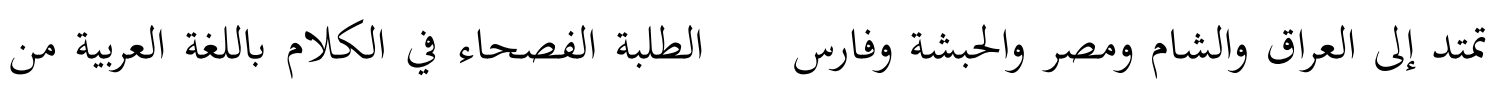

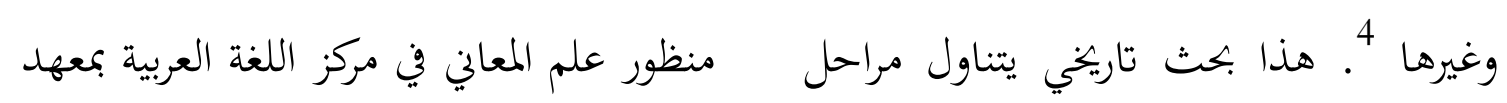

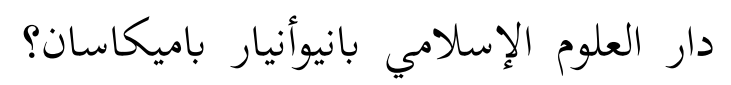
تطور اللغة العربية الفصحى.

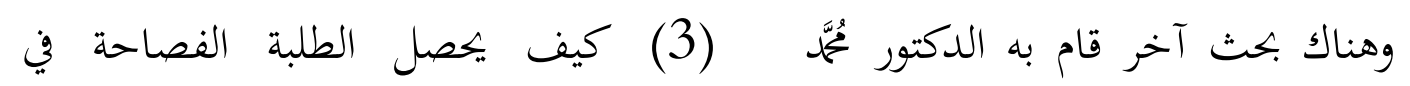
الحباس بعنوان "كلمات في ميزان الفصاحة الكام الكلام باللغة العربية من منظور علم المعاني 
استخدامه في بجال الظواهر الطبيعية المختلفة المختده

ولمعرفة الواقع الحالي لمواصفات الفصاحة عند الطلبة الفصحاء في مركز اللغة العربية بمعهد دار العلوم الاسلامي قام الباحثان بجمع البيانات بأداة الاستبانة والمقابلة والملاحظة. والاستبانة تتكون من بـان سؤالين: واحد يتعلق بسؤال البحث الأول وامدال

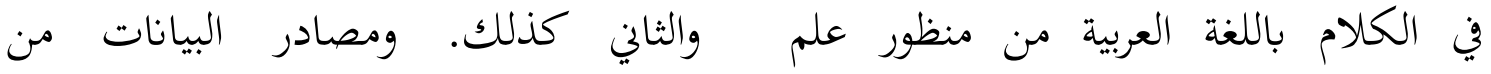
الأساتذة في مركز اللغة العربية بمعهد بانيوأنيار وكان عددهم عشرين أستاذا.

ج. الإطار النظري

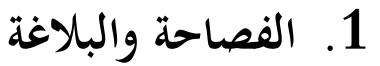

الفصاحة لغة البيان والظهور، قال الله تعالى: "وأخي هَارونُ هو أفصحُ مني" لهُ أي واني أبينُ مني منطقا وأظهر مني قولا. 9 والفصاحة

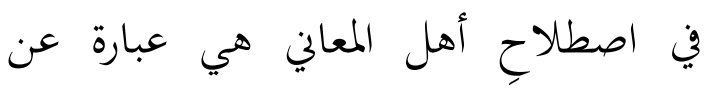
الألفاظ البينة الظاهرة، المتبادرة إلى الفهم، الفئ والمأنوسة الاستعمال بين الكتّاب والشعراء لمكان حسنها. فالفصاحة تشمل الكلمة،

; D. Sugiono, 220 خوقان عبيدات، عبد الرمن عدس، و كايد عبد الحق، "Metode Penelitian", Bandung: Alfa Beta, 2000, 9 والنشر والتوزيع، 1997), 3264 ج جني 1.
في مركز اللغة العبية بمعهد دار العلوم الإسلامي بانيوأنيار باميكاسان؟ ومن هتين الأسئلتين تستهدف هذئ بئن الدراسة إلى ما يلي: (1) معرفة وجود الطلبة الفصحاء في الكلام باللغة العربية من منظور علم المعاني في مركز اللغة العربية بمعهد دار العلوم الإسلامي بانيوأنيار باميكاسان. (2) معرفة أهم مواصفات الطلبة الفصحاء المعاني في مركز اللغة العربية بمعهد دار العلوم الإسلامي بانيوأنيار باميكاسان. (2) معرفة طريقة حصول الطلبة الفصاحة في الكلام باللغة العربية من منظور علم المعاني في مركز اللغة العربية بمعهد دار العلوم الإسلامي

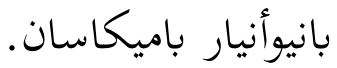

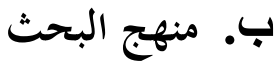
تستخدم هذه الدراسة المنهج النوعي بالأسلوب الوصفي في معرفة وجود الطلبة الفصحاء ومعرفة أهم مواصفاتم في منظور علم المعاني. والأسلوب الوصفي مرتبط منذ نشأته بدراسة المشكلات المتعلقة بالمجالات

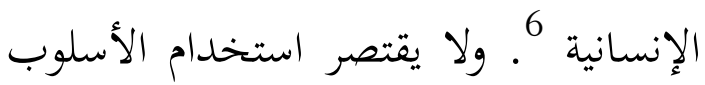

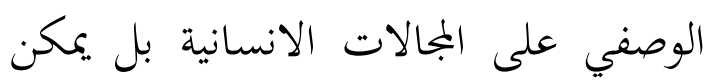
6 ذوقان عبيدات، عبد الرحمن عدس، و كايد عبد الحق، البحث العلمي: مغهومه أدواته

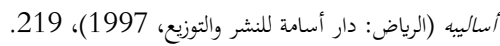


في مُشَنَّ ومُرْمَلِ) فقد وصفها بكثرة الشعر والتفافه. 13

وأما الغرابة فهي كون الكلمة غير

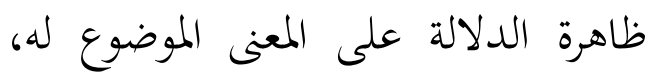

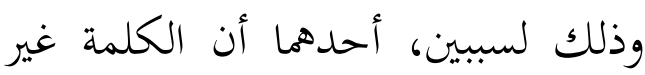
متداولة في لغة العرب، فيحتاج لمعرفة معناها الرجوع إلى المعاجم والقواميس، مثال ذلك قول عيسى بن عمرو النحوي إلى المايسي وقد سقط عن دابته فالتنّ حوله الناسئ

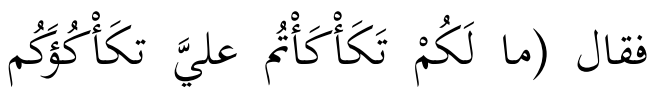

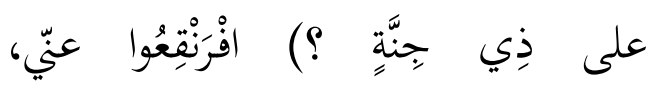

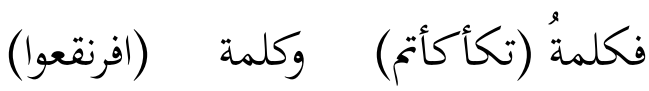
غريبتان، أي مالكم اجتَمَعْتم تنشُُوا عَّيّ.

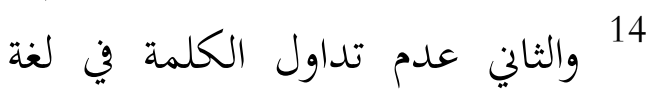
العرب الشائعة، كلفظ مسَّرج من قول رؤبة بن العجاج (ومقلة وحاجبا مزججا

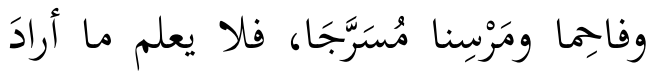
بقوله مسرَّجا حتى اختلف أئمة اللغة في

$$
\text { تخريجه } 15
$$

وأما مخالفة الوضع فهو كون الكلمة خخالفة لما ثبت معناه عند علماء اللغة
والكلام، والمتكلم، فيقال كلمة فصيحة، وكلام فصيح، ومتكلم فصيح. أ) فصاحةُ الكلمة تكون الكلمة فصيحة إذا كانت

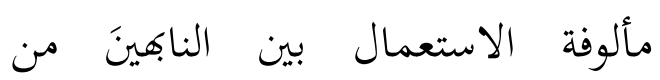

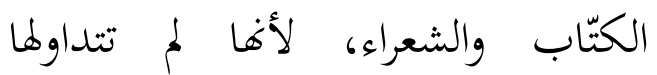

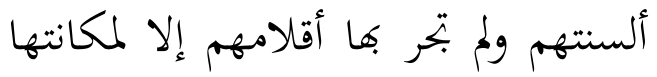
من الحسن باستكمالها عناصر الجودة، وصفاتِ الجمالِ. فشروط فصاحة الكلمة

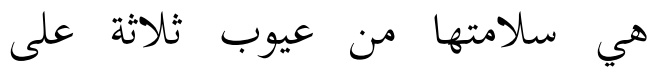
الأقل، وهي تنافر الحروف والغرابة خخالفة

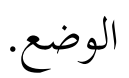
أما تنافرُ الحروف فهو ثقل الكلمة عند وقعها على السمع وصعوبة أدائها

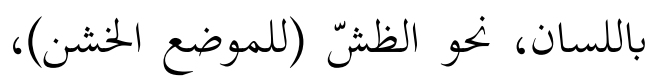

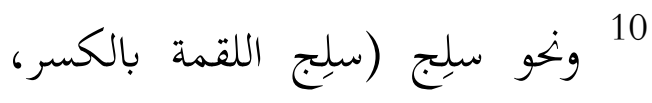
يسلجها سلجا وسلجانا، أي بلعها)

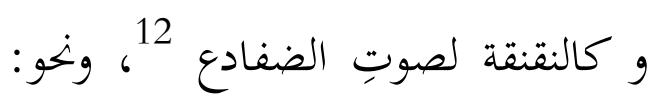
مستشزرات بمعنى مرتفعات من قول امرئ القيس يصف شُعر ابنة عمه (غَادئرئه

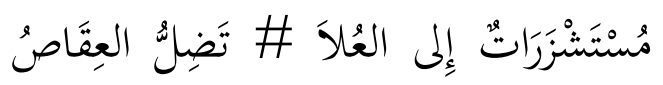


المشهورة، كأن يكون الكلام جاريا على خلاف ما اشتهر من قوانين النحو

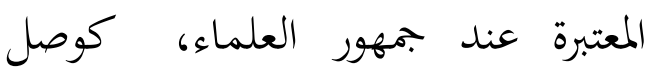
الضميرين، وتقديم غير الأعراف منهما

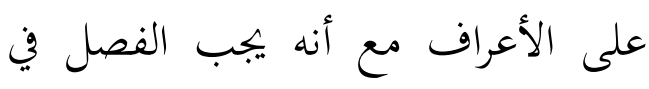
تلك الحالة، كقول الشاعر (جَزَى بَنُوهُ أبا

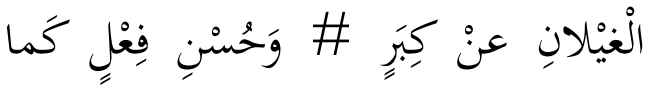

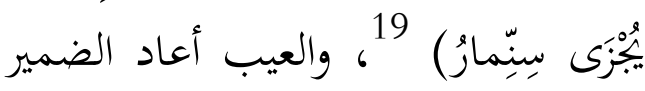
في بنوه على أبي غيلان وهو متأخر لفظا ورتبة، لأنه مفعول به، ورتبته التأخير.

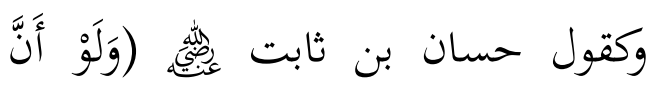

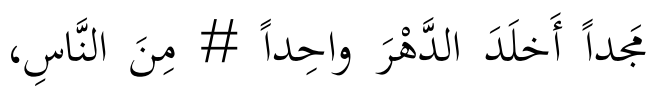

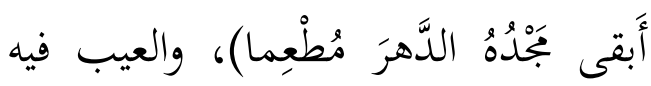
أن الشاعر أعاد الضمير في مجده على الى لئل مطعم، وهو متأخر لفظا ورتبة، لأنه

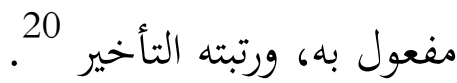
والثالث التعقيد، وهو نوعان التعقيد اللفظي والتعقيد المعنوي: التعقيد اللفظي هو أن يكون الكلام خفي الدلالة على الكي المعنى المراد به بحيث تكون الألفاظ غير مُرتبة على وفق ترتيبٍ المعاني. وينشأ ذلك التعقيد من تقديم أو تأخير أو فصل

\footnotetext{
19 أحمد القلقشندي, صبح الأعشى في كتابة الإنشاء، 291 ج 1. 20مصطفي الغلاييني، جامع الدروس العربية (بيروت: المكتبة العصرية، 1994)، 149
}

مثل الأجلل في قول أبي النجم (الحَمْدُ

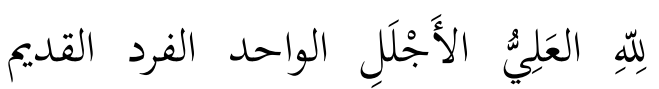
الأوَّل) فإن القياس الأجلّ بالإدغام، و العالِ لا مسوّغ لفكّة، فهو يريد الأجل وأظهر الإديان التضعيف ضرورة ل. 16. ب) فصاحةُ الكلام تكون فصاحة الكلام بسلامته من عيوب ثلاثة هي تنافر الكلمات وضعف

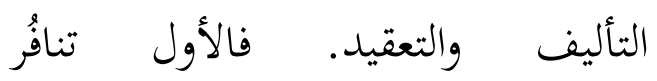
الكلمات، فلا يكونُ اتصال بعضها ببعض مما يسبب ثقلها على السمع،

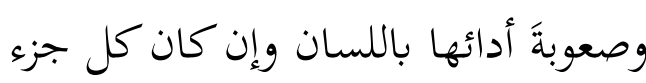
منها على انفراده فصيحا كالشطرِ الثاني

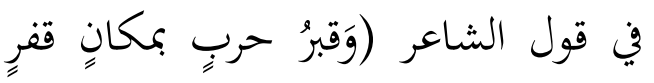

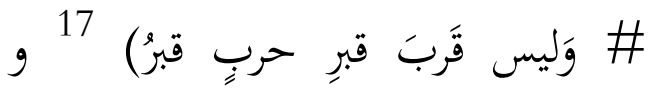
كالشطرِ الأولِ في قول أبي تُّام (كَرِيٌ

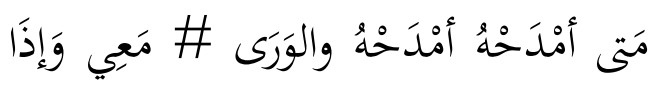

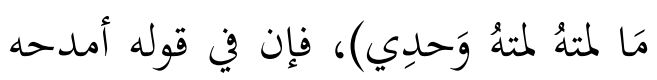

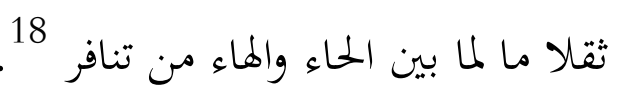
والثاني ضعفُ التأليفِ، وهو خروج الكلام عن قواعد اللغة المطردة

\footnotetext{
16 الخطيب القزويني مُحَّة بن عبد الرحمن جلال الدين، الإيضاح في علوم البلاغة (بيروت:

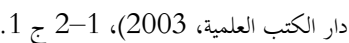
17 الخطيب القزويني يُمَّمُ بن عبد الرحمن جلال الدين, الإيضاح في علوم البلاغة.

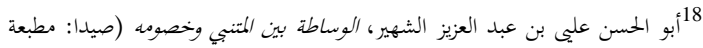

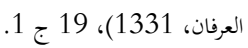




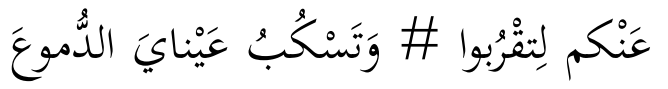

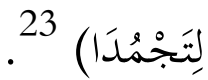

جعل سكب الدُموع كناية عما يلزم في فراق الأحبة من الحزن والكمد، فأحسنَ وأصاب في ذلك، ولكنه أخطأ في جعل جمود العين كناية عما يوجبه التّلاقي من الفرح والسرور بقُرب أحبته، وهو خفى وبعيد إذ لم يعرف في كلام العرب عند الدعاء لشخص بالسرور، أنْ

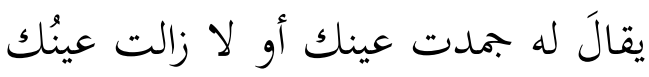
جامدة، بل المعروف عندهم أن جمود العين إنما يكنى به عن عدم البكاء حالة المعاد الحزن، كما في قول الحنساء (أَعَيَيَّ جُجودا

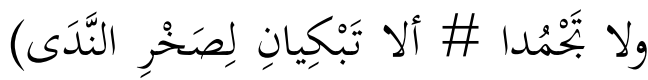

ج) فصاحة المتكلم

هي عبارة عنِ الملكة التي يقتدر بها صاحبها على التعبير عن المقصود بكلام فصيح، في أي غرض كان 25 في فيكون النعير عن المعود بكلام قادرا بصفة الفصاحة الثابتة في نفسه

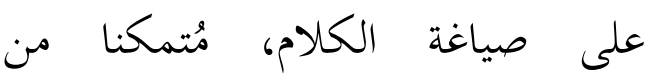

بأجنبي بين الكلمات التي يجب أن تتجاور ويتصل بعضها ببعض كقول

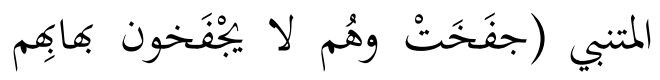
\# شيَمّ على الحسَب الأغبرِ دلائلُ). أصل جفخت افتخرت بمم شيمَ دلائل على الحسب الأغر هم لا يجفخون بما 21

ومثل (ما قرأ إلا واحدا مُمَّمَ مع كتابا أخيه) كان هذا الكلام غير فصيح، لأن فيه تعقيدا لفظيا، ولكن التعبير الفصيح هو (ما قرأ تُمَّمَ مع أخيه إلا كتابيا واحدا). التعقيد المعنوي هو أن يكون الكلام

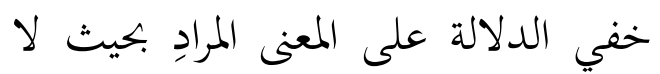
يفهم معناه إلا بعد عناء وتفكير طويل.

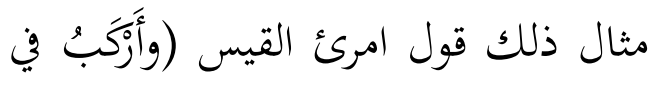

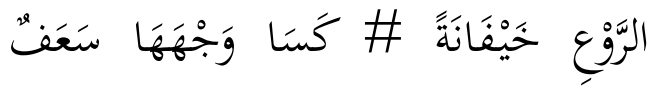

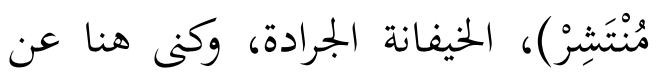

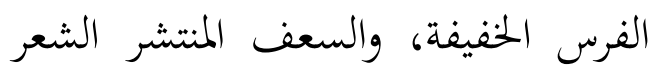
يكسو وجهها فقبيحُ 22 وكما في قول المتشر الشعبر عباس بن الأحنف (مَأطلُبُ بُعْدَ الدَّارِ

\footnotetext{
23 أمد القلقشندي، صبح الأعشى في كتابة الإنشاء، 292 ج 1. 24 صدر الدين علي بن أبي الفرج بن الحسن البصري، الحماسة البصرية (القاهرة: مكتبة

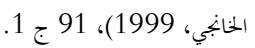
25 أحمد الهاشيمي، جواهر البلاعة في المعاني والبيان والبديع، 30.
} 
اعتقدوا أفم يعرفون القضية حسن المعرفة لأغم قد شاركوا الطلبة في المعهد وأشرفوا عليهم اثناء تعلمهم اللغة العربية خاصة في مبنى خاص يسمى بمركز اللغة العربية. ويقصد بمركز اللغة العربية في معهد دار العلوم الاسلامي بانيوأنيار باميكاسان هو مؤسسة تربوية تابعة لمعهد دار العلوم الاسلامي بانيوأنيار غير المدرسة تقوم بتعليم اللغة العربية في بيئة خاصة داخل مبنى معين منه، ويسكن فيه عدد من طلبة المعهد بعد أن نجحوا في اختبار القبول الذي عقد في آخر شهر مايو لكل سنة دراسية 27. وتكون مدة الدراسة في هذا المركز سنة

واحدة حيث تبدأ من أول يونيو وتنتهي إلى

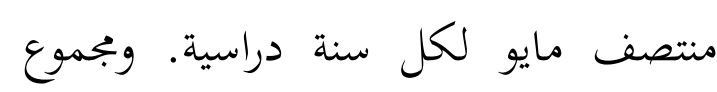
لأيام للسنة الدراسية في هذا المركز 211 يوما تقديرا باعتبارها الأيام الدراسية بعد الغاء العطلة الأسبوعية واجازة المعهد السنوية وغيرها

وتنفذ الأنشطة التعليمية لكل صباح قدر ساعة واحدة (60 دقيقة) من السادسة إلى السابعة. والأنشطة الأخرى بعد صلاة

27 27. المادي، "المنهج على أساس المواقف في مركز اللغة العربية بعمهد دار العلوم

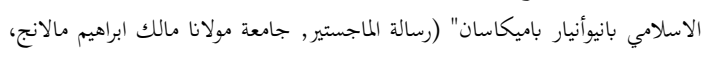
نور المادي.
التصرف في ضُروبه، بصيرا بالخوض في جهاته ومناحيه 26. وهذه النظرية هي كالأساس الذي يرجع اليه هذه الدراسة. وجدير بالذكر أن هناك المصطلحات متساوية اللفظ قريبة المعنى غير المقصود في هذه الدراسة، مثل اللغة الفصيحة أو الفصحى والفصاحة في القراءة عند القراء. وأما اللغة الفصيحة فيقصد بها اللغة المعتبرة عند العرب وهي ضد اللغة العامية أو السوقية التي تنششر في كلام العرب في الشوارع والشوق. وتكون اللغة الفصيحة لغة صحيحة من حيث القواعد والمعنى الصرفي و والمعجمي. وأما الفصحية في القراءة عند القراء فهي مطابقة اللفظ بمخارج الحروف و وأحكام

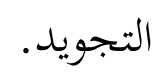
د. نتيبجة البحث 1 ـ مركز اللغة العربية لقد أجرى الباحثان هذه الدراسة واختارا مصادر البيانات من الأساتذة الذين كانوا من مشرفي مركز اللغة العربية في معهد دار العلوم بانيوأنيار باميكاسان؛ عيث

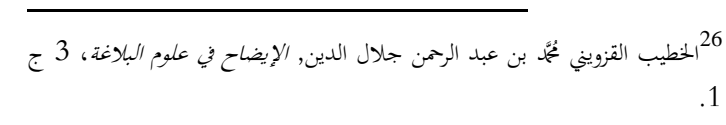


على الكلام باللغة العربية ويهدد من تركها

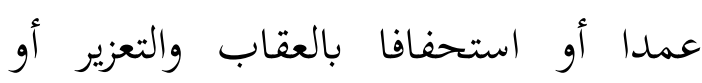

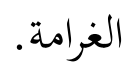

فبهذا النظام أصبح مركز اللغة العربية بيئة تعين على تكوين ألسنتهم على النطق باللغة العربية الفصحى كما يدعمهم في لئن استعياب الغة العربية لفهم دين الاسلام والقرآن الكريم 30

2. - 2. - الطلبة الفصحاء

من البيانات التي جمعها الباحث على

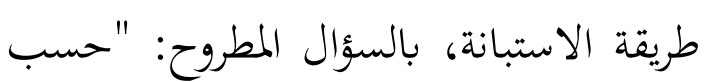
خبرتك في تعليم اللغة العربية واشرافها في مركز اللغة العربية بمعهد دار العلوم بانيوأنيار باميكاسان لكل الفترة الدراسية التي تكون سنة واحدا، هل تجد من الطلبة الفصحاء

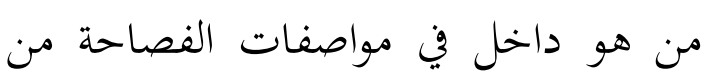
منظور علم المعاني؟ - من داخ

فمن هذا السؤال، أجاب الأساتذة نهو 95\% عن وجود الطلبة الفصحاء لكل هل هل فترة. وأستاذ واحد فقط أو ما يساوي 50 \% يجيب أن كون الطلبة الفصحاء يتعلق بشخصية الطلبة أنفسهم، بمعنى أنه ليس لئب أبعاء

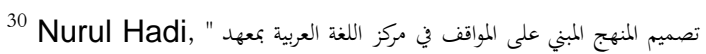
"دار العوم بانيوأنيار باميكاسان" Okara\&58; Jurnal Bahasa dan Sastra 9, 181-154 عدان 2 (2015).
العشاء قدر نصف الساعة (من السابعة ليلا إلى السابعة والنصف) لتقديم حفظ المفردات اليومي والتدرييات، تم تنفيذ المناقشة عن الأحكام الشرعية باللغة العربية حوالي ساعة

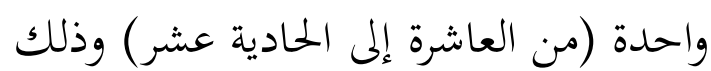
بعد مشاركتهم في تعلم كتب التراث التابع للمعهد. وهذه الأنشطة التعليمية تستمر كل يوم وليلة إلا يوم الجمعة، وأما ليلة الجمعة فتعقد فيها المحاضرة العامة من المحاضر المدعو من خارج المعهد 29. يتكون مبنى المركز من أربع غرف، المعن، واحدة منها مخصص للمكتب الإداري ويسكن فيه مدير المركز وبعض المشرفين، وثلاث غرف باقية يسكن فيها طلاب المركز مع رعاية بعض المشرفين كرؤساء الغرف. وأمام تلك الغرف الأربعة قاعة كبيرة مكانا لاجراء الأنشطة. وتستخدم اللغة العربية الفصحى في جميع الأنشطة المركزية وفي المعاملة اليومية بين

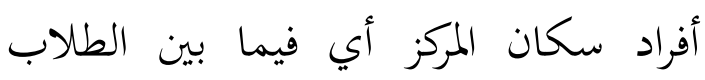

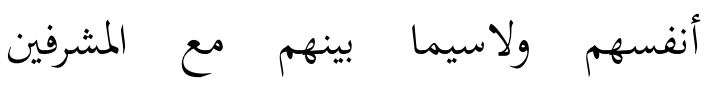
والأساتذة. ويوم الثلاثاء والجمعة ولياليهما يسمى بيوم العربية حيث يجبر طلاب المركز 
مفروضا أن يكون موجودا في كل سنة الكلام، مُمتمكنا من التصرف في ضُروبه،

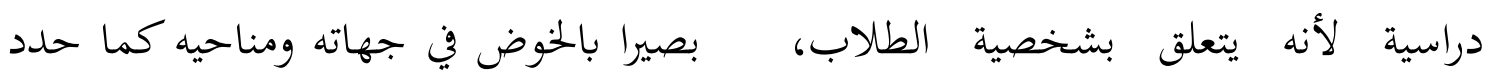

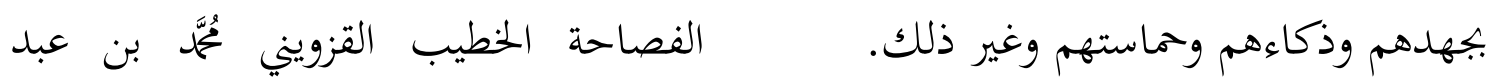
الرممن جلال الدين.

فوجد الباحث البيانات التالية:

أ) حصل الصفة الأولى وهي ملكة الطلبة في القاء الخاضرة أو الحطبة باللغة العربية في موضوعات مختلفة عشرين في المائة ب)

ب) وحصل الصفة الثانية وهي ملكة الطلبة

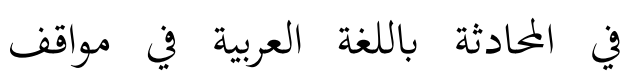

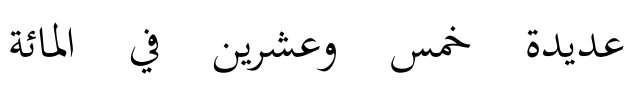

ج) وحصل الصفة الثالثة وهي ملكة الطلبة في مشاركة مسابقة الجدال باللغة العربية في موضوعات كثيرة أربعين في المائة (\% $\%$ (\%)

د) وحصل الصفة الرابعة وهي قدرة الطلبة

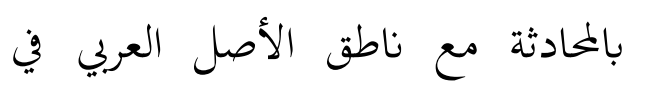
مواقف متنوعة خمس وعشرين في المائة

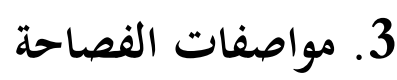

وأما مواصفات الفصاحة عند الطلبة

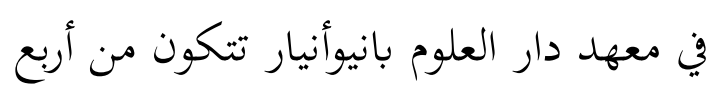
صغات:

أ) لدى الطلبة الملكة في القاء المحاضرة أو

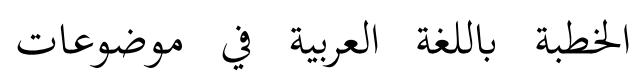

مختلفة.

ب) لدى الطلبة الملكة في المحادثة باللغة العربية في مواقف عديدة. ج) لدى الطلبة الملكة في مشاركة مسابقة الجدال باللغة العربية في موضوعات الطاتية كثيرة. د) لدى الطلبة القدرة بالمحادثة مع ناطق الأصل العربي في مواقف متنوعة.

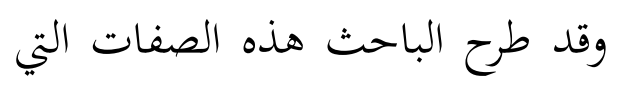
تدل على فصاحة الطلبة في المعهد من ملن الصغات الئ

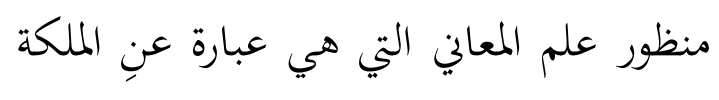

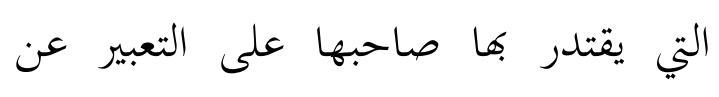

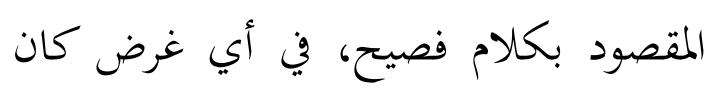

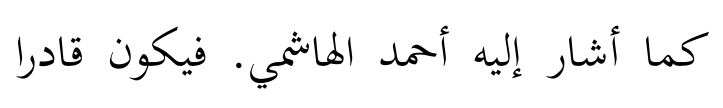
بصفة الفصاحة الثابتة في نفسه على صياغة آلماند 
قدر على المشاركة في هذه المسابقة ولاسيما

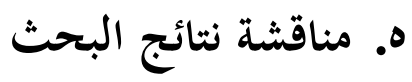
حصل على الفوز والجائزة فهو داخل في فئي الفصاحة من منظور علم المعاني.

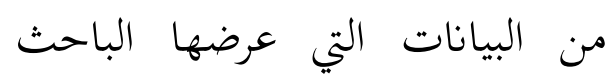
السابقة تبين أن الطلبة الفصحاء في معهد البيات والصفة الأقوي التالية هي ملكور المعاني دار العلوم بانيوأنيار متواجدون في كل فترة. الطلبة في المحادثة باللغة العربية في مواقف الفيه ولكن العدد قليل لأن الفصاحة في الكلام

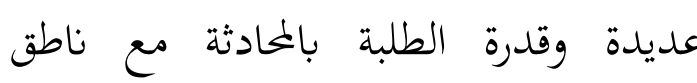
باللغة العربية تتعلق بالشخصية. فالطلبة

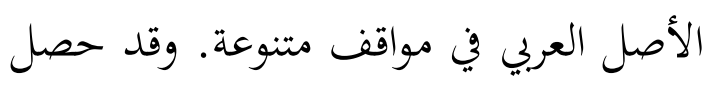
كل من هذه الصفة خمس وعشرين في المائة

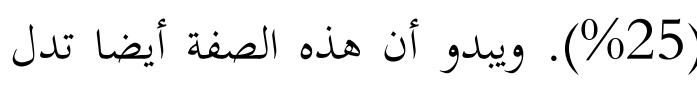

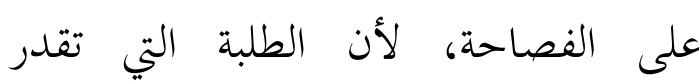
بالمحادثة باللغة العربية في مواقف عديدة يعني

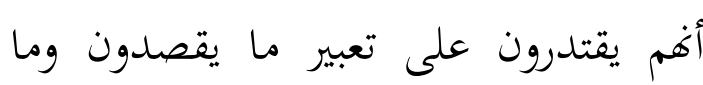

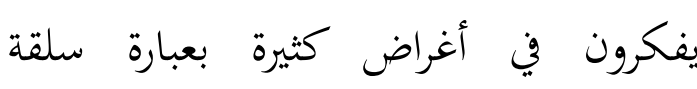
ومفهومة. ولاسيما أغم قادرون على المحادثة عع الناطقين باللغة العربية الأصليين، فهذا دلالة قوية على الفصاحة.

وأدنى الصفة للفصاحة عند الطلبة هي ملكتهم في القاء المحاضرة أو الخطبة باللغة العربية في موضوعات مختلفة حيث حصلت على عشرين في المائة (10\%). رغم أن القاء الحطبة باللغة العربية في موضوعات كثيرة هي من أقوى صفات الفصاحة عند بلدية العرب القديم ولكنه قليل من الطلبة الناطقين

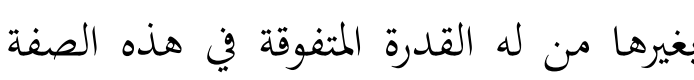

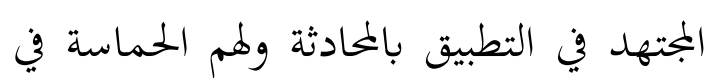
اكتساب وتعلم اللغة العربية ولهم الذكاء القوي فهؤلاء الذين حصلوا بصفة الفصاحة. بل اعترف بعض الأساتذة أنه قد تحدث باللغة العربية بعد ما تعلم اللغة لعربية في مركز اللغة خلال أربع أشهر فقط. فهذا يدل على أن الطلبة في مركز اللغة العربية في معهد دار العلوم بانيوأنيار بعد تعلمهم اللغة العربية سنة كاملة -طبعا- موجود من هم الفصحاء في الكلام باللغة العربية. وأما الصفة التي تختص بفصاحة الطلبة في الكالام باللغة العربية في معهد بانيوأنيار تبينت من البيانات المعروضة أن أقوي الصفة هي ملكة الطلبة في مشاركة مسابقة الجدال باللغة العربية في موضوعات كثيرة حيث حصلت أربعين في المائة (40\%). وهذا معقول لأن مسابقة الجدال باللغة العربية تحتاج إلى كفاءة التعبير الشفهي العالية. فمن 
التطبيق بالمحادثة ولهم الحماسة في اكتساب وتعلم اللغة العربية ولهم الذكاء

القوي فهؤلاء الذين حصلوا على الفصاحة في الكلام باللغة العربية.

ويعتقد الباحث أن مواصفات

الفصاحة عند الطلبة التي وجدها في معهد دار العلوم بانيوأنيار الاسلامي هي كذلك موجودة في المعاهد الاسلامي الأخرى التي لهي طبق تعليم اللغة العربية المكشفة مثل مركز اللغة العربية في ذلك المعهد.

\section{قائمة المراجع}

أبو الحسن عليى بن عبد العزيز الشهير. الوساطة بين المتنبي وخصومه. صيدا: مطبعة العرفان، 1331.

أبو نصر إسماعيل بن حماد الجوهري الفارابي. الصحاح تاج اللغة وصحاح العربية. بيروت: دار العلم للمايين، 1987. أحمد القلقشندي. صبح الأعشى في كتابة الإنشاء. القاهرة: دار الكتب المصرية،

أحمد الهاشمي. جواهر البلاعة في المعاني والبيان والبلديع. الطبعة الثانية عشرة.
رغم أفم فصحاء في الكلام أو المحادثة. فالخطبة هي الملكة العالية المتميزة لا يملكها إلا قليل ولاسيما القاءها باللغة العربية.

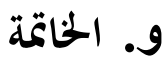

وفي الختام يستنتج الباحث من

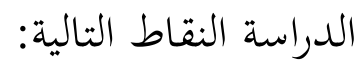
1. أن الطلبة الفصحاء في الكلام باللغة العربية متواجدون في معهد دار العلوم بانيوأنيار الاسلامي باميكاسان، بل

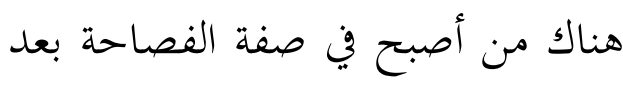
أربعة أشهر بعد دراستهم اللغة العربية في مركز اللغة العربية. 2. أن هناك أربع صفات للطلبة الفصحاء، مرحه العريه، وأقوى الصفات هي ملكة الطلبة في مشاركة مسابقة الجدال باللغة العربية في

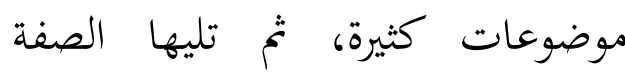
"ملكة الطلبة في المحادثة باللغة العربية في المواقف العديدة والقدرة على المحادثة مع الناطقين بالعربية الأصليين وأدنى الصفة هي الملكة في القاء الخطبة باللغة العربية في موضوعات مختلفة. 3. أن كيفية حصول الطلبة الفصاحة في الكلام باللغة العربية تتعلق بشخصية كل منهم أنفسهم. فالطالب المجتهد في بلئل 
صدر الدين علي بن أبي الفرج بن الحسن البصري. الحماسة البصرية. القاهرة:

مكتبة الخانجي، 1999.

ضياء الدين ابن الأثير، المثل السائر في أدب

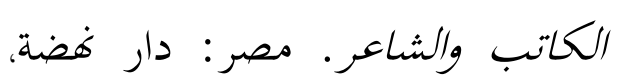

.د.

عمــار، علـي عمـــار علـي. "مراحل تطور اللغة العربية الفصحى". قراءات عات عاسل 1 1 عدد 1 (30 أبريل، 2008):

$$
.96-79
$$

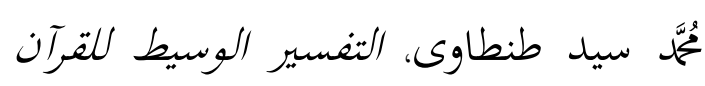

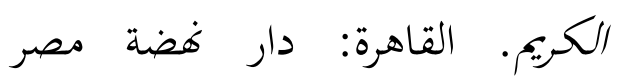

$$
\text { للطباعة والنشر والتوزيع، } 1997 .
$$

مصطفي الغلاييني. جامع الدروس العربية.

بيروت: المكتبة العصرية، 1994.

نور المادي. "المنهج على أساس المواقف في مركز اللغة العربية بعمهد دار العلوم الاسلامي بانيوأنيار باميكاسان". رسالة الماجستير، جامعة مولانا مالك بالك

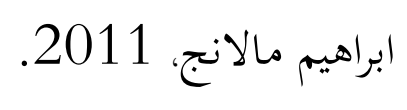

Effendy, Ahmad Fuad. "Metodologi Pengajaran Bahasa Arab". Malang: Misykat, 2005.
إندزنيسيا: مكتبة دار احياء الكتب

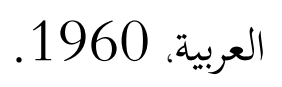

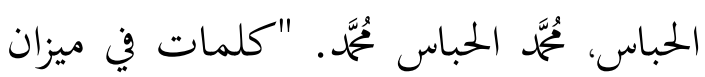
الفصاحة العربية". اللّنة العربية 9 عدد 2 (1 أكتوبر، 2007): 53.82

الخطيب القزويني يُمَّمَ بن عبد الرمن جلال الدين. الإيضاح في علوم البلاغة. بيروت: دار الكتب العلمية، 2003. الفوزان، عبد الرحمن بن إبراهيه, مختار الطاهر

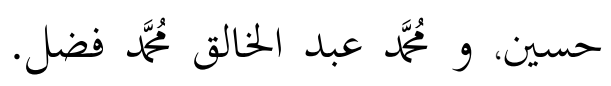
العربية بين يديك كتاب الطالبج1. العربية للجميع، 2007. القرآن العظيم، د.ت. جمال الدين يُمَّمَ بن مكرم. لسان العرب. بيروت: دار صادر, د.ت. ذوقان عبيدات، عبد الرمن عدس، و كايد

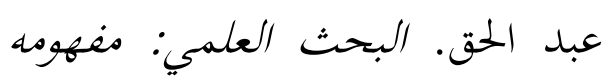
أدواته أساليبه. الرياض: داضئ السئ أسامة للنشر والتوزيع، 1997. سيد تُمَّمَ مرتضى الحسيني الزبيدي. تاج العروس من جواهر القاموس. كويت: مطبعة الحكومة، 1965. 
تصميم المنهج المبني على " Hadi, Nurul. 181.

المواقف في مركز اللغة العربية بمعهد دار

Sugiono, D. "Metode Penelitian".

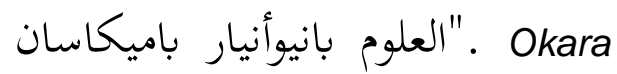
Bandung: Alfa Beta, 2000. \& 58; Jurnal Bahasa dan Sastra 